Old Dominion University

ODU Digital Commons

Biological Sciences Faculty Publications

Biological Sciences

2007

\title{
Mutations in Transmembrane Domains 1, 4 and 9 of the Plasmodium falciparum Chloroquine Resistance Transporter Alter Susceptibility to Chloroquine, Quinine and Quinidine
}

\author{
Roland A. Cooper \\ Old Dominion University \\ Kristan D. Lane \\ Old Dominion University \\ Bingbing Deng \\ Jianbing $\mathrm{Mu}$ \\ Jigar J. Patel
}

See next page for additional authors

Follow this and additional works at: https://digitalcommons.odu.edu/biology_fac_pubs

Part of the Biochemistry Commons, $\underline{\text { Microbiology Commons, and the Molecular Biology }}$ Commons

\section{Repository Citation}

Cooper, Roland A.; Lane, Kristan D.; Deng, Bingbing; Mu, Jianbing; Patel, Jigar J.; Wellems, Thomas E.; Su, Xinzhuan; and Ferdig, Michael T., "Mutations in Transmembrane Domains 1, 4 and 9 of the Plasmodium falciparum Chloroquine Resistance Transporter Alter Susceptibility to Chloroquine, Quinine and Quinidine" (2007). Biological Sciences Faculty Publications. 282.

https://digitalcommons.odu.edu/biology_fac_pubs/282

\section{Original Publication Citation}

Cooper, R. A., Lane, K. D., Deng, B. B., Mu, J. B., Patel, J. J., Wellems, T. E., ... Ferdig, M. T. (2007). Mutations in transmembrane domains 1, 4 and 9 of the Plasmodium falciparum chloroquine resistance transporter alter susceptibility to chloroquine, quinine and quinidine. Molecular Microbiology, 63(1), 270-282. doi:10.1111/j.1365-2958.2006.05511.x 
Authors

Roland A. Cooper, Kristan D. Lane, Bingbing Deng, Jianbing Mu, Jigar J. Patel, Thomas E. Wellems, Xinzhuan $\mathrm{Su}$, and Michael T. Ferdig 


\section{Mutations in transmembrane domains 1, 4 and 9 of the Plasmodium falciparum chloroquine resistance transporter alter susceptibility to chloroquine, quinine and quinidine}

Roland A. Cooper, ${ }^{1 *}$ Kristin D. Lane, ${ }^{1}$ Bingbing Deng, ${ }^{2}$ Jianbing Mu, ${ }^{3}$ Jigar J. Patel, ${ }^{2}$

Thomas E. Wellems, ${ }^{3}$ Xinzhuan $\mathrm{Su}^{3}$ and

Michael T. Ferdig ${ }^{2}$

${ }^{1}$ Department of Biological Sciences, Old Dominion

University, Norfolk, VA 23529, USA.

${ }^{2}$ Department of Biological Sciences, University of Notre Dame, Notre Dame, IN 46556, USA.

${ }^{3}$ Laboratory of Malaria and Vector Research, National Institute of Allergy and Infectious Diseases, Bethesda, MD 20892, USA.

\section{Summary}

Mutations in the Plasmodium falciparum chloroquine (CQ) resistance transporter (PfCRT) can result in verapamil-reversible $C Q$ resistance and altered susceptibility to other antimalarials. PfCRT contains 10 membrane-spanning domains and is found in the digestive vacuole (DV) membrane of intraerythrocytic parasites. The mechanism by which PfCRT mediates $C Q$ resistance is unclear although it is associated with decreased accumulation of drug within the DV. On the permissive background of the $P$. falciparum 106/1 ${ }^{\mathrm{k} 76}$ parasite line, we used singlestep drug selection to generate isogenic clones containing unique pfcrt point mutations that resulted in amino acid changes in PfCRT transmembrane domains 1 (C72R, K76N, K76I and K76T) and 9 (Q352K, Q352R). The resulting changes of charge and hydropathy affected quantitative $C Q$ susceptibility and accumulation as well as the stereospecific responses to quinine and quinidine. These results, together with a previously described S163R mutation in transmembrane domain 4 , indicate that transmembrane segments 1,4 and 9 of PfCRT provide important structural components of a substrate recognition and translocation domain. Charge-affecting mutations within these segments may affect the ability of PfCRT to bind different

Accepted 31 October, 2006. *For correspondence. E-mail rcooper@ odu.edu; Tel. (+1) 757683 4755; Fax (+1) 7576835283. quinoline drugs and determine their net accumulation in the DV.

Introduction

The continuing burden of malaria has been aggravated by the evolution and spread of Plasmodium falciparum resistant to chloroquine (CQ) (Baird, 2005). Despite recent advances in understanding the genetic basis for resistance in $P$. falciparum, the molecular mechanism remains uncertain. $C Q$ accumulates in the parasite digestive vacuole (DV), a lysosome-like organelle where host haemoglobin is digested to amino acids during the intraerythrocytic stage of infection (Yayon et al., 1985; Goldberg et al., 1990). Free haematin is released as a toxic by-product of haemoglobin breakdown and is normally sequestered as an inert microcrystalline form known as haemozoin (Pagola et al., 2000). In the DV, CQ and other quinolines are believed to bind haematin and inhibit the formation of haemozoin (Sullivan et al., 1996), resulting in the build-up of lethal drug-haematin complexes (Chou and Fitch, 1980; Dorn et al., 1998).

A series of genetic mapping and allelic association studies has shown that $\mathrm{CQ}$ resistance (CQR) arose by mutations in the $P$. falciparum chloroquine resistance transporter (PfCRT) protein (Fidock et al., 2000; Djimde et al., 2001; Wootton et al., 2002). A putative member of the drug/metabolite transporter (DMT) superfamily (Martin and Kirk, 2004; Tran and Saier, 2004), PfCRT contains 10 predicted transmembrane domains (TMDs) and is localized to the DV membrane of trophozoite-stage parasites (Cooper et al., 2002). Although various haplotypes of PfCRT exist in naturally CQ-resistant parasite populations, all forms show changes in at least 4 of 15 different amino acid positions (Bray et al., 2005). Every such parasite has so far been found to carry the charge-loss mutation K76T, or, in a single documented case, $\mathrm{K} 76 \mathrm{~N}$ (Huaman et al., 2004; Cooper et al., 2005). No other mutation is completely associated with CQR on a global scale, raising the question of the relative contribution of other mutations to resistance per se, versus their role in balancing endogenous protein function and accommodating the charge loss. PfCRT mutations are also thought to 
Table 1. Effect of PfCRT mutations in the $106 / 1^{\mathrm{K} 76}$ line of $P$. falciparum on susceptibility to CQ, QN and QD, alone and in the presence of VP.*

\begin{tabular}{|c|c|c|c|c|c|c|c|c|}
\hline \multirow[b]{2}{*}{ Drug } & \multicolumn{8}{|c|}{ Parasite line } \\
\hline & $\mathrm{Dd} 2^{\dagger}$ & $106 / 1^{\mathrm{K} 76}$ & $106 / 1^{76 \mathrm{~N} \ddagger}$ & $106 / 1^{76 T \ddagger}$ & $106 / 1^{761 \ddagger}$ & $106 / 1^{72 R-761 \S}$ & $106 / 1^{761-352 K \S}$ & $106 / 1^{761-352 \mathrm{R} \S}$ \\
\hline$C Q$ & $383 \pm 17$ & $34 \pm 1.3$ & $304 \pm 13^{n}$ & $445 \pm 11^{\pi}$ & $589 \pm 19^{\Uparrow 1}$ & $34 \pm 1.0^{* *}$ & $26 \pm 0.7^{* *}$ & $50 \pm 2.2^{* *}$ \\
\hline$C Q+V P$ & $41 \pm 1.4$ & $30 \pm 0.9$ & $39 \pm 1.5$ & $35 \pm 0.8$ & $41 \pm 1.2$ & $38 \pm 1.0$ & $28 \pm 1.2$ & $34 \pm 1.8$ \\
\hline QN & $324 \pm 13$ & $205 \pm 7.8$ & $349 \pm 11^{\pi}$ & $382 \pm 22^{\Uparrow}$ & $16 \pm 4.7^{\Uparrow}$ & $168 \pm 10^{* *}$ & $240 \pm 6.8^{* *}$ & $170 \pm 9.1^{* *}$ \\
\hline $\mathrm{QN}+\mathrm{VP}$ & $52 \pm 1.3$ & $201 \pm 8.4$ & $39 \pm 3.2$ & $70 \pm 5.0$ & $73 \pm 4.5$ & $187 \pm 7.0$ & $206 \pm 7.2$ & $98 \pm 8.3$ \\
\hline QD & $187 \pm 8.0$ & $57 \pm 5.6$ & $65 \pm 4.9$ & $112 \pm 6.3^{\Uparrow}$ & $89 \pm 4.2^{\uparrow}$ & $52 \pm 3.8^{\star \star}$ & $68 \pm 3.1^{\text {** }}$ & $48 \pm 2.5^{\star *}$ \\
\hline $\mathrm{QD}+\mathrm{VP}$ & $16 \pm 5.0$ & $55 \pm 5.1$ & $12 \pm 1.1$ & $14 \pm 1.0$ & $25 \pm 5.1$ & $50 \pm 3.4$ & $57 \pm 3.3$ & $33 \pm 2.2$ \\
\hline
\end{tabular}

${ }^{*} \mathrm{C}_{50}$ values $(\mathrm{nM})$ from $\left[{ }^{3} \mathrm{H}\right]$-hypoxanthine incorporation measurements are reported as means \pm SEM from 4-18 independent assays. VP was used at a fixed concentration of $0.9 \mu \mathrm{M}$.

tControl CQ-resistant line.

挫es selected with CQ pressure from 106/1 176 .

sines selected with QN pressure from 106/1761.

"Mean $I_{50}$ value significantly different from $106 / 1^{\mathrm{K} 76}$ parent line $(P<0.05)$.

${ }^{* *}$ Mean $I C_{50}$ value significantly different from $106 / 1^{761}$ parent line $(P<0.05)$.

affect cross-resistance and the potency of structurally diverse drugs that target the DV (Cooper et al., 2002; Sidhu et al., 2002; Ferdig et al., 2004; Johnson et al., 2004).

Models to explain CQR hinge on the observed decrease in drug accumulation associated with mutant PfCRT. Both sensitive and resistant parasites retain an equal capacity for CQ-haematin binding, yet resistant parasites have developed a mechanism to reduce $\mathrm{CQ}$ accumulation at its site of action in the DV (Bray et al., 1998; Saliba et al., 1998). Some reports have suggested that altered drug accumulation results from an energydependent CQ efflux mechanism (Krogstad et al., 1992; Sanchez et al., 2004; 2005; Naude et al., 2005); others have suggested passive efflux of diprotic CQ out of the DV by a 'charged drug leak' mechanism (Warhurst, 2003; Johnson et al., 2004; Bray et al., 2006). Experiments using a yeast heterologous expression system have shown that direct CQ-PfCRT binding may play a role in altered CQ accumulation (Zhang et al., 2004).

The $106 / 1^{\mathrm{K} 76}$ line of $P$. falciparum is a CQ-sensitive isolate containing six of seven PfCRT mutations characteristic of many Old World CQ-resistant isolates (M74IN75E-A220S-Q271E-N326S-R372I), lacking only the critical K76T mutation (Fidock et al., 2000). Earlier, we reported the emergence of CQ-resistant parasites containing novel $\mathrm{K} 76 \mathrm{~N}$ and $\mathrm{K} 76 \mathrm{I}$ PfCRT mutations from $106 / 1^{\mathrm{K} 76}$ following single-step selection with $C Q$, demonstrating a key role for the loss of the charged $\mathrm{K} 76$ residue in the resistance mechanism (Cooper et al., 2002). We also observed that mutant parasites carrying the K76I mutation (106/1 ${ }^{761}$ line) exhibited stereoselective changes in response to the isomers quinine (QN) and quinidine (QD), suggesting drug-PfCRT binding as a component in the mechanism of action of these drugs.

Here we report further results on the molecular basis of PfCRT-mediated drug selectivity and sensitivity. We have used single-step QN pressure to generate a panel of resistant clones from the 106/1 ${ }^{761}$ line, each carrying a single-point mutation resulting in an amino acid change in PfCRT. In each case, gain of a critical lysine or arginine residue was observed at a unique position in a TMD of the PfCRT protein. We also demonstrate under single-step $\mathrm{CQ}$ selection the emergence of the PfCRT K76T substitution ubiquitous in CQR field isolates. Quantitative changes in drug potency, stereoselectivity and $C Q$ accumulation were associated with these mutations. Our findings implicate direct drug-PfCRT interactions in CQR and in altered susceptibility to QN and QD.

\section{Results Selection with QN results in novel PfCRT second-site
suppressor mutations}

Our previous pharmacological characterization of a 106/ $1^{761}$ mutant showed that this highly CQ-resistant line exhibits an unusual degree of QN sensitivity $\left(\mathrm{IC}_{50} 16 \mathrm{nM}\right.$; Table 1) (Cooper et al., 2002). To explore the effect of PfCRT mutations on this sensitivity, we subjected 106/1 ${ }^{761}$ parasites to additional single-step selection experiments with $100 \mathrm{nM}$ QN (Fig. 1A). These experiments produced three populations of QN-resistant parasites that, when evaluated by DNA sequencing, exhibited different novel PfCRT mutations: C72R, Q352K and Q352R (lines designated 106/1 $1^{72 R-761}, \quad 106 / 1^{761-352 K}$ and 106/1 ${ }^{761-352 R}$ respectively). No changes other than these mutations were detected in the pfort or pfmdr1 sequences, and analysis using the multicopy microsatellite marker PfRRM (Su et al., 1998) confirmed that each clone was of identical provenance with the 106/1761 (and also 106/1 ${ }^{\mathrm{K} 6}$ ) line (data not shown). The codon changes at position 72 (TGC to $\mathrm{CGC}$ ) and position 352 (CAA to AAA or CGA) produced amino acid changes in predicted TMD 1 or TMD 9 of PfCRT respectively. 
A

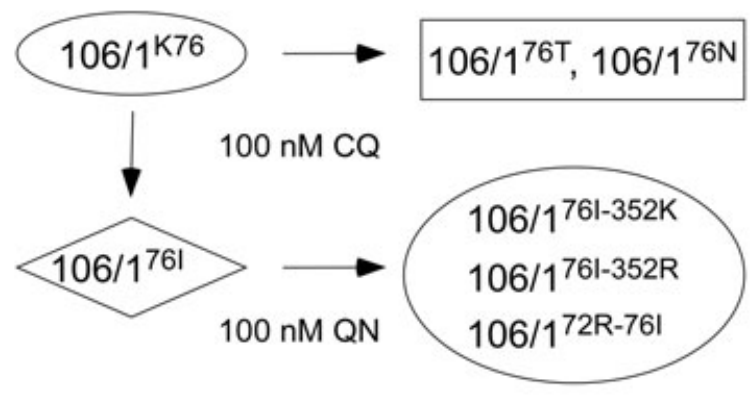

\section{$\bigcirc$ CQ-sensitive, QN-resistant clones \\ $\square$ CQ-resistant, QN-resistant clones \\ $\diamond$ CQ-resistant, QN-sensitive clone}
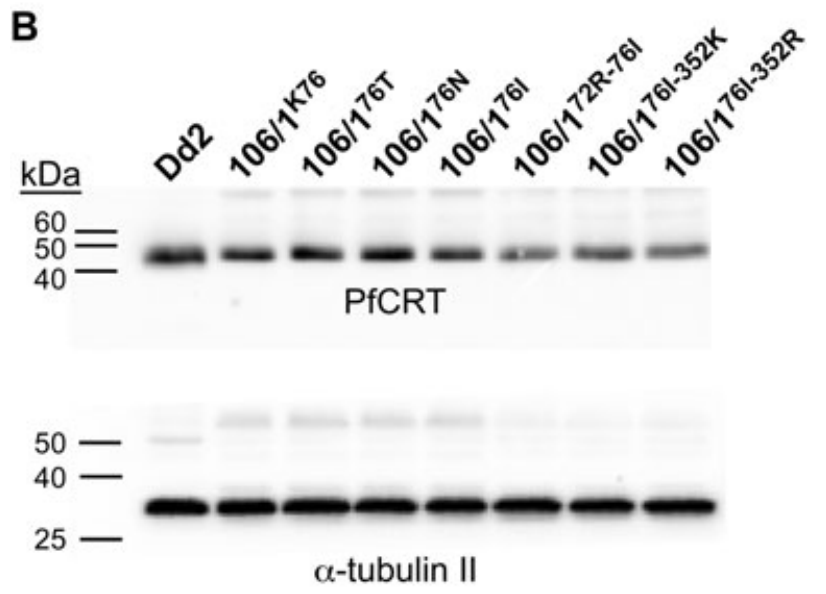

Fig. 1. Selection pathway of PfCRT mutants and immunoblot analysis.

A. Selection of PfCRT mutant parasite lines from the 106/1 176 and $106 / 1^{761}$ line. Mutations were selected by subjecting $10^{9}-10^{10}$ parasites to single-step $100 \mathrm{nM} \mathrm{CQ}\left(106 / 1^{\mathrm{k} 76}\right)$ or QN $\left(106 / 1^{761}\right)$ pressure.

B. Immunoblots show similar PfCRT expression levels in the mutant parasite lines relative to $106 / 1^{\mathrm{K} 76}$. Lower blot is a loading control, showing similar levels of $P$. falciparum $\alpha$-tubulin. Protein from equal numbers of synchronous trophozoites was separated on $12 \%$ SDS-PAGE and transferred to PVDF membrane. PfCRT and $\alpha$-tubulin II were detected by chemiluminescence using anti-PfCRT or anti- $\alpha$-tubulin II antibody. Molecular weight $(\mathrm{kDa})$ ladders are illustrated on the side of the membrane.

Multiple immunoblots of parasite lysates were performed to compare expression levels of PfCRT from parental and derived mutant lines (Fig. 1B). These immunoblots showed small differences in PfCRT levels from experiment to experiment, but the differences varied in pattern among the lines and did not associate with drug response. Similar results are reported from fresh field isolates (Durrand et al., 2004). We attribute these observations to slight differences in the stage of the parasites at harvesting.
Drug assays of the $106 / 1^{72 R-761}, 106 / 1^{761-352 K}$ and 106/ $1^{761-352 R}$ lines confirmed their greatly reduced sensitivities to QN relative to $106 / 1^{161}$ parasites (Fig. 2A). Mean Hill slope values derived from curve fit data of all QN-selected lines were significantly steeper than that of the parent $106 / 1^{761}$ line and differed from each other as well ( $F$-test; $P<0.05)$, suggesting a change in binding cooperativity between QN and the different forms of PfCRT (data not shown). We note that inferring a specific molecular mechanism based on slope change is difficult because of the complexities in associating PfCRT-drug interactions with an assay outcome of $\left[{ }^{3} \mathrm{H}\right]$-hypoxanthine incorporation as a surrogate for cell death.

Drug assays showed the QN-selected lines had returned to full $C Q$ sensitivity (CQS) and had lost the inverse relationship of stereospecific QN and QD potencies we reported previously for the $106 / 1^{761}$ parasite (Table 1) (Cooper et al., 2002). The restoration of CQS in a CQ-resistant line by an arginine substitution at position 72, or an arginine or a lysine substitution at position 352, provides evidence for the importance of a critical positive charge in PfCRT TMD 1 or 9 for CQS in addition to TMD 4 reported earlier (Johnson et al., 2004). We note that $106 / 1^{761-352 R}$ has an elevated CQ IC 50 value $(50 \mathrm{nM})$ for a CQ-sensitive line and retains $30-40 \%$ verapamil (VP) reversibility against $C Q$ and $Q N$, suggestive of an intermediate CQR phenotype (Table 1).

In parallel with the above work, we also performed single-step selection experiments with CQ on the 106/1 ${ }^{\mathrm{K} 76}$ line (Fig. 1A). These experiments led to recovery of parasites with the PfCRT K76T mutation (106/176T line) in addition to the $106 / 1^{761}$ and $106 / 1^{76 \mathrm{~N}}$ lines reported earlier (Cooper et al., 2002) (Table 1). In common with the QN-selected mutant lines, sequencing and genotyping confirmed that the 106/1 ${ }^{76 \mathrm{~T}}$ line was derived from 106/1 ${ }^{\mathrm{K} 76}$ and carried only one new mutation in pfcrt, and no changes in the pfmdr1 sequence. Similarly, expression levels of mutant PfCRT were comparable to that of 106/1 ${ }^{\mathrm{K} 76}$ (Fig. 1B). Consistent with all CQ-resistant field isolates, mutant $106 / 1^{76 T}$ parasites displayed the VP-reversible CQR phenotype (Table 1). Figure 2B illustrates the rightward shift of the sigmoidal CQ doseresponse curve that occurred with the K76T mutation. The $\mathrm{CQ}$ dose-response curves from all three selected CQ-resistant lines had slightly shallower, but statistically significant changes $(F$-test; $P<0.05)$ in the Hill coefficients compared with $106 / 1^{\mathrm{K} 76}$, and differed from each other as well (data not shown). These slope changes suggest that the mutations again resulted in altered binding cooperativity, in this case between PfCRT and CQ. Relative to $106 / 1^{\mathrm{K} 76}, 106 / 1^{76 \mathrm{~T}}$ parasites also showed significant decreases in QN and QD sensitivity, and onset of VP sensitization (Table 1), demonstrating a critical role of the PfCRT K76T field mutation in reduced sensitivity to 

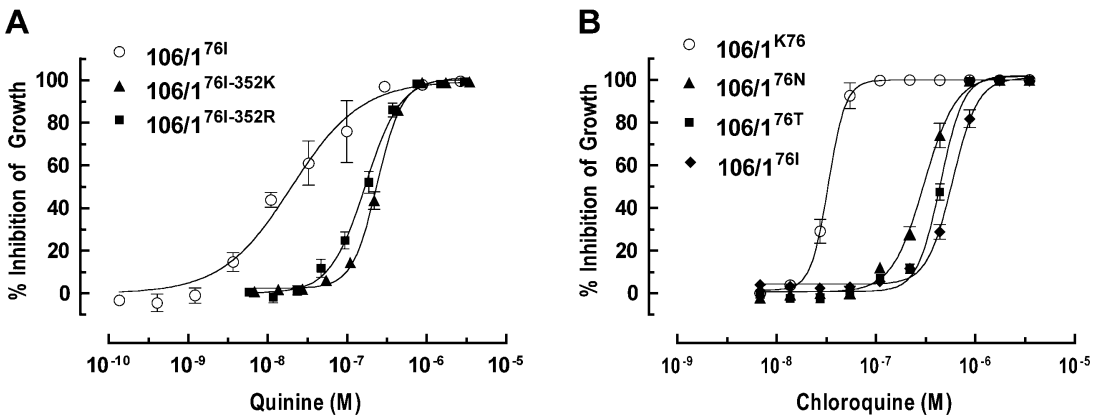

Fig. 2. PfCRT position 76 and 352 mutations determine specific levels of $Q N$ and $C Q$ susceptibility.

A. Changes in sensitivity to QN following selection of a Q352K or Q352R mutation within PfCRT TMD 9 in the 106/1761 line. Data points on the dose-response curves from $\left[{ }^{3} \mathrm{H}\right]$-hypoxanthine incorporation assays represent mean \pm SEM from at least four independent experiments. Mean $\mathrm{IC}_{50}$ values and Hill slopes derived from curve fitting were significantly different for each mutant $(P<0.5)$. Data for the QN-selected line $106 / 1^{72 R-761}$ (Table 1 ) is not illustrated for clarity.

B. Dose-response curves illustrating the changes in CQ sensitivity following selection of K76N, K76T or K76I mutations in PfCRT TMD 1 of the $106 / 1^{\mathrm{K} 76}$ line. Data points represent mean \pm SEM of at least four independent experiments. Mean IC 50 and Hill slope values were significantly different for the curve fit from each mutant $(P<0.5)$.

multiple drugs. The susceptibilities of all mutant parasite lines to the structurally unrelated sesquiterpene lactone artemisinin were also determined. Similar to our earlier observations (Cooper et al., 2002), small changes were observed within the low nanomolar range. No trends were observed between artemisinin sensitivity and specific pfort mutations (data not shown). Finally, we have observed that the drug susceptibility profiles of all pfort mutant lines have remained stable after continuous culture for many months in the absence of any drug pressure (data not shown).

\section{Ability of parasites to accumulate $C Q$ is associated with PfCRT mutations}

CQ-resistant lines of $P$. falciparum limit the concentration of $\mathrm{CQ}$ in their DVs (Krogstad et al., 1987). In confirmation of this association, CQ-resistant lines $106 / 1^{76 T}$ and $106 / 1^{761}$ accumulate far less drug than the original CQ-sensitive $106 / 1^{\mathrm{K} 76}$ line; Fig. 3 shows the relative accumulations reduced by approximately 22- and eightfold, respectively, in the $1 \mathrm{~h}$ assay conditions of the present study. With reversion of the mutant $106 / 1^{761}$ lines to $\mathrm{CQ}$-sensitive phenotypes, $\mathrm{CQ}$ accumulation was increased again but not to the levels of the original $106 / 1^{\mathrm{K} 76}$ line: parasites 106/1 ${ }^{761-352 R}, 106 / 1^{72 R-761}$ and 106/ $1^{761-352 \mathrm{~K}}$ each showed greater $\left[{ }^{3} \mathrm{H}\right] \mathrm{CQ}$ mean counts than their $106 / 1^{761}$ parent CQ-resistant line (Fig. 3); of these, however, only counts from the $106 / 1^{761-352 K}$ line were found to differ significantly from those of the parent line by $t$-test $(P<0.05)$. Although the correlation between $\mathrm{CQ}$ $\mathrm{IC}_{50}$ values and accumulation of $\left[{ }^{3} \mathrm{H}\right] \mathrm{CQ}$ may not be linear among the lines tested, an overall relationship between these two parameters is apparent. The trend for a small twofold accumulation increase for $106 / 1^{761-352 R}$ would also be consistent with its residual phenotype of CQR noted above.

\section{VP competitively inhibits the $Q N$ response of $106 / 1^{761}$}

In addition to its unusual degree of susceptibility to QN, $106 / 1^{761}$ is the only reported line of $P$. falciparum against which QN is more potent than its diastereomer, QD (Cooper et al., 2002) (Table 1). The reversal agent VP normally chemosensitizes CQ-resistant parasites to $\mathrm{CQ}$, QN and QD (Kyle et al., 1990). However, VP surprisingly acts as a QN inhibitor (increasing its $I_{50}$ ) in 106/1 ${ }^{761}$, despite potentiating the response to its isomer $\mathrm{QD}$, as well as to $\mathrm{CQ}$, in the normal reported fashion (Table 1). To

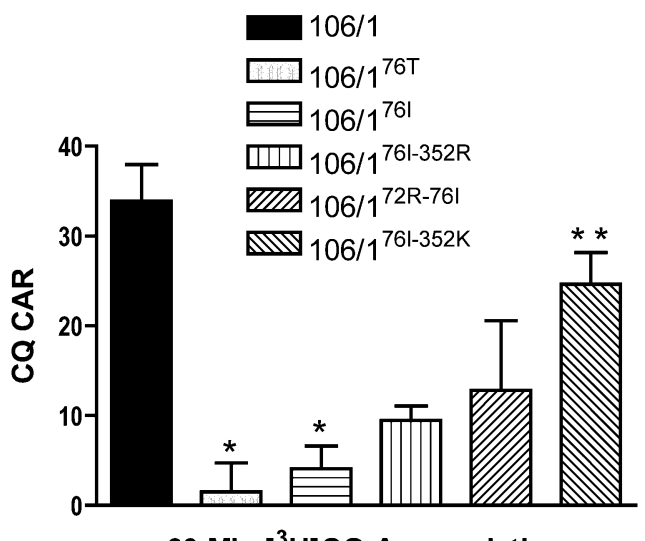

60 Min $\left[{ }^{3} \mathrm{H}\right] \mathrm{CQ}$ Accumulation

Fig. 3. PfCRT mutations determine parasite $C Q$ accumulation. Mutations at positions 72, 76 and 352 of PfCRT affect the CQ cellular accumulation ratio (CAR). Trophozoite-infected erythrocytes were incubated in the presence of $50 \mathrm{nM}\left[{ }^{3} \mathrm{H}\right] \mathrm{CQ}$ for $60 \mathrm{~min}$. Single asterisks indicate CAR differs significantly from the $106 / 1^{\mathrm{K} 76}$ parent $(P<0.05)$. Double asterisks indicate CAR differs significantly from the $106 / 1^{761}$ parent $(P<0.05)$. 

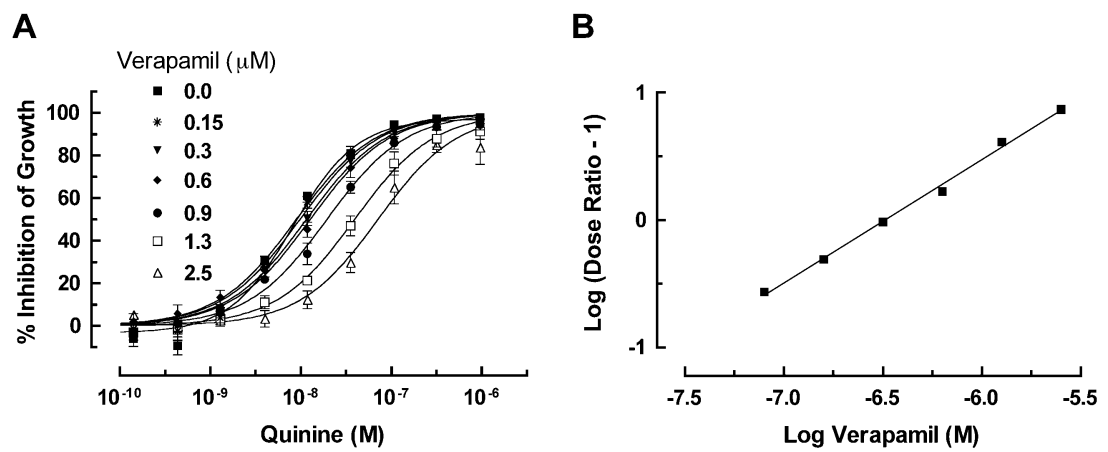

Fig. 4. Schild regression indicates a competitive interaction between QN and VP in the $106 / 1^{761}$ line.

A. Changes in QN dose-response curves in the presence of different concentrations of VP. Shifts to the right in the dose-response curves highlight the inhibitory effect of VP against QN. Values represent means \pm SEM from three independent assays.

B. Linear Schild regression plot derived from dose-response data. The slope is not significantly different from unity $(0.9689$; $95 \%$ confidence interval $=0.8833-1.054$ ), indicating a simple competitive interaction between QN and VP. The X-intercept predicts an equilibrium dissociation constant $\left(K_{\mathrm{B}}\right)$ of $324 \mathrm{nM}(95 \%$ confidence interval $=289-358 \mathrm{nM})$ for VP with its binding site.

further investigate the nature of this unique interaction, we performed Schild analysis on the antagonistic properties of VP against QN in the 106/176l line. Schild analysis tests whether the rightward shifts in agonist (QN) doseresponse curves in the presence of incremental concentrations of antagonist (VP) fit a model of simple competition between the two drugs for a common binding or receptor site (Arunlakshana and Schild, 1959). A Schild regression slope not significantly different from unity indicates simple competition. Under these assumptions, the $K_{\mathrm{B}}$, or antagonist equilibrium dissociation constant, is estimated from the regression $\mathrm{X}$-intercept. Schild analysis is mathematically applicable only to the principles of drug antagonism (Arunlakshana and Schild, 1959) and thus was not used to study the potentiating effect of VP on CQ in this and other parasite lines. We measured QN susceptibility in a series of dose-response tests against 106/1761 in the presence of six concentrations of VP (Fig. 4A). Regression fitting of the Schild data produced a linear plot with a slope of unity $[0.97 ; 95 \%$ confidence interval $(\mathrm{Cl})$ of $0.88-1.05]$, pointing to a competitive interaction between QN and VP. The X-intercept predicts a dissociation constant $\left(K_{\mathrm{B}}\right)$ of $324 \mathrm{nM}(95 \% \mathrm{Cl}$ of $289-358 \mathrm{nM})$ for VP and its binding site (Fig. 4B). Combined with observations of the stereospecific drug effect on QN and QD, these data suggest a site of competitive interaction between $Q N$ and VP is present at PfCRT in the $106 / 1^{761}$ line.

\section{PfCRT position 76 mutations alter interactions in quinoline combinations}

Dose combinations consisting of $\mathrm{CQ}$ and $\mathrm{QN}$ are reported to act antagonistically in P. falciparum, both on parasite morphology and in terms of fractional IC $\mathrm{C}_{50}$ values (Stahel et al., 1988; Fitch, 2004). To evaluate the influence of PfCRT mutations on quinoline interactions, we tested the effects of $C Q, Q N$ and $Q D$ in pair-wise combinations and plotted the fractional $I C_{50}$ data as isobolograms (Berenbaum, 1978). The CQ:QN combination was slightly synergistic in the parent $106 / 1^{\mathrm{K} 76}$ line, slightly antagonistic in $106 / 1^{76 T}$ and clearly synergistic against $106 / 1^{76}$, reflecting the unusual QN sensitivity in this line (Fig. 5). In contrast, the CQ:QD pair was antagonistic in all parasite lines, regardless of PfCRT haplotype, indicating differences in the molecular interactions with $Q N$ versus $Q D$.

Stereoisomer drug pairs usually bind to the same receptor molecule, but often with greatly different affinities (Laduron, 1988; Triggle, 1996). Wesche and Black (1990) demonstrated an additive interaction between the cinchona alkaloid stereoisomer pairs in CQ-sensitive and $C Q$-resistant lines of $P$. falciparum. Similarly, in our assays, the QN:QD combination was additive to slightly synergistic with respect to $106 / 1^{\mathrm{K} 76}$ and $106 / 1^{76 \mathrm{~T}}$ (Fig. 5); isobolograms for $106 / 1^{16 \mathrm{~N}}$ were qualitatively similar to those of the $106 / 1^{76 T}$ line (data not shown). In the $106 / 1^{761}$ line, however, QN:QD was synergistic, indicative of a major and unique effect of the position 76 isoleucine. Yet this effect is context-specific, as isobol trends for all double mutants derived from $106 / 1^{761}$ resembled the original CQ-sensitive parent, 106/1 ${ }^{\mathrm{K} 76}$ (data not shown). The additional selection of arginine at PfCRT position 72, or to lysine or arginine at position 352, abrogated the effects of the position 76 isoleucine on drug interactions.

$C Q I C_{50}$ values correlate with charge change and amino acid hydropathy at PfCRT position 76

An earlier bioinformatic analysis predicted that parasite susceptibility to desethylamodiaquine and the extent of VP chemosensitization to $C Q$ would relate to the hydropathy of mutable PfCRT amino acids (Warhurst, 2003). To test this prediction, we examined the relationship of $C Q$ 

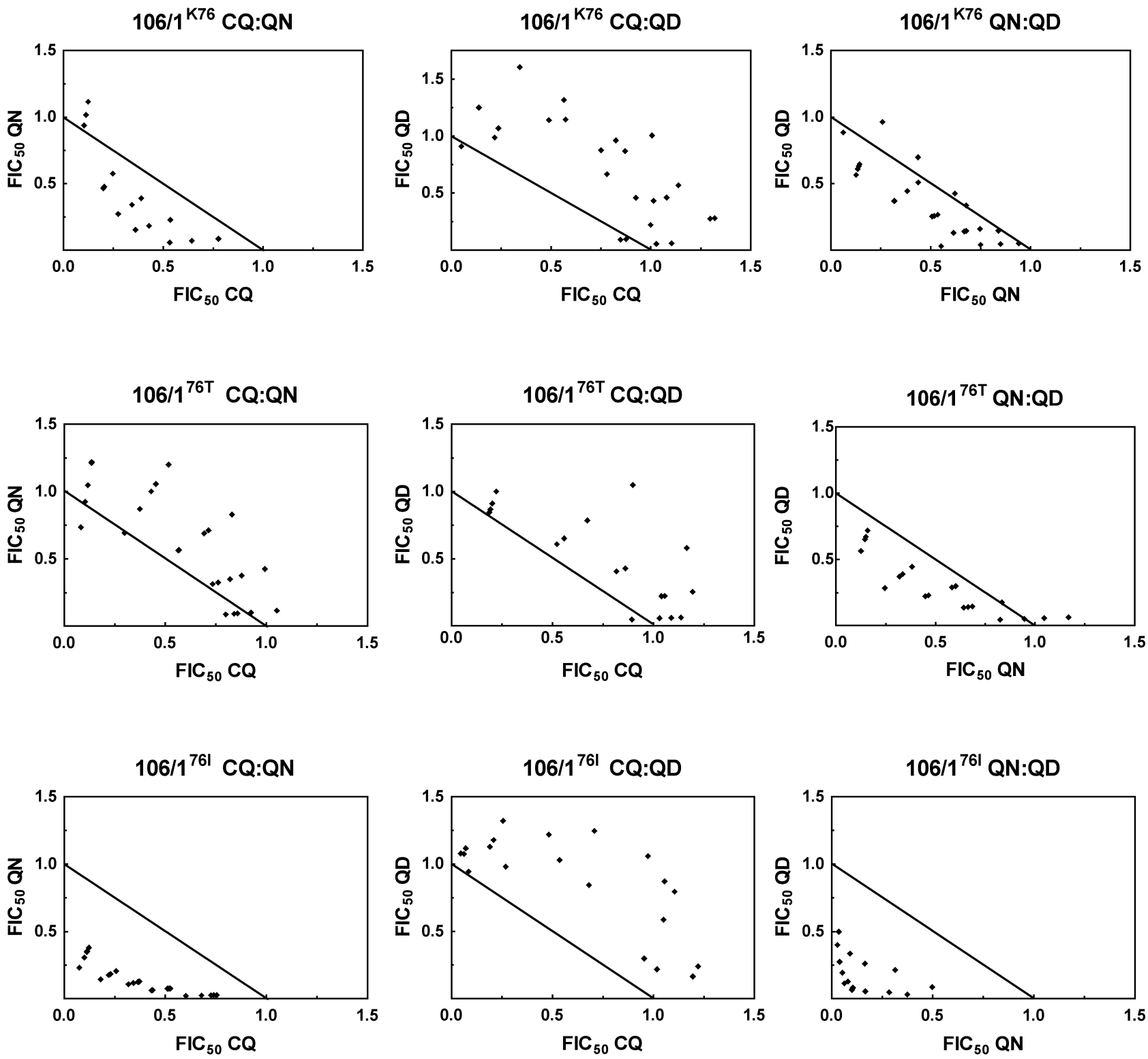

Fig. 5. The PfCRT K76I mutation alters quinoline drug interactions. Isobolograms illustrating the effects of amino acid changes at PfCRT position 76 on quinoline drug combinations in the 106/1 ${ }^{\mathrm{K} 76}$ line of $P$. falciparum. Data points represent the fractional $I C_{50}$ values of various dose combinations of $C Q: Q N, C Q: Q D$ and QN:QD. Points near the solid line indicate an additive relationship between the two drugs, those above the line indicate antagonism and those the below the line indicate a synergistic relationship. The plotted data show that interactions involving QN become highly synergistic following a K76I mutation. Each isobologram was derived from a series of three independent dose-response experiments; data points from all experiments are shown.

$\mathrm{IC}_{50}$ values to hydropathy at position 76 as an indication of the hydrophobic and hydrophilic tendencies of these amino acids to affect drug susceptibility. Figure $6 \mathrm{~A}$ shows a sharp increase in $C Q I C_{50}$ value with the loss of charge and slight change in hydropathy corresponding to the lysine to asparagine substitution at position 76 . The $I C_{50}$ values of the CQ-resistant mutants track with the increasing hydropathies of asparagine, threonine and isoleucine respectively. We discerned no relationship between $\mathrm{CQ} \mathrm{IC}_{50}$ values and side-chain volumes of the different amino acids at position 76 (Fig. 6B). In a similar analysis, no associations between amino acid 76 hydropathy or volume and QN or QD susceptibilities were detected (data not shown).

\section{Discussion}

Genetic linkage studies have shown that point mutations in the pfcrt gene of $P$. falciparum determine VP-reversible CQR as well as specific susceptibilities and cross- 

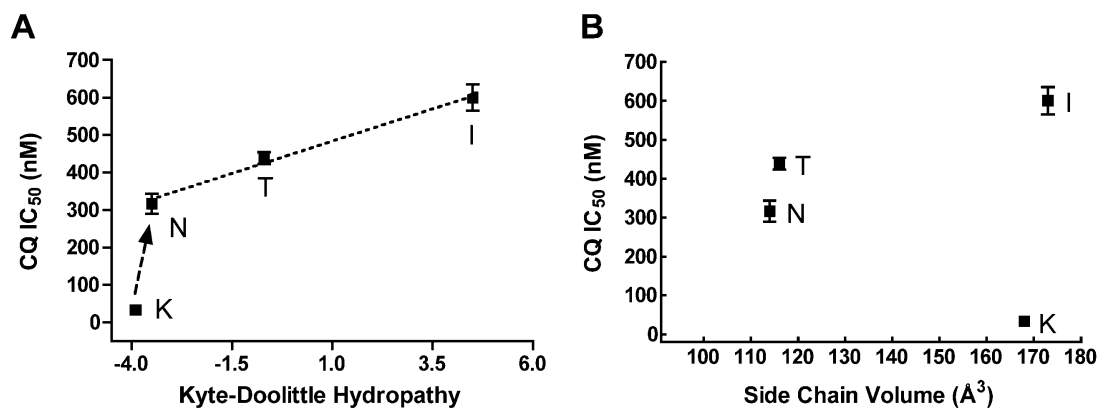

Fig. 6. $\mathrm{CQ}$ resistance correlates with charge loss and hydropathy of PfCRT position 76 residues.

A. CQ IC 50 plotted as a function of Kyle-Doolittle hydropathy. Mean $C Q I C_{50}$ values are significantly different between all four lines $(P<0.05$; unpaired $t$-tests; Table 1), and slope for the regression of $\mathrm{N}$, T and I versus $\mathrm{CQ} \mathrm{IC} 50$ is non-zero $(P<0.05)$. Letter abbreviations for the corresponding amino acids are shown adjacent to the data points on the graph.

B. No relationship was observed between mean $\mathrm{CQ} \mathrm{IC}_{50}$ values and side-chain volume of the position 76 amino acids.

resistance of this parasite to other quinoline antimalarials (Fidock et al., 2000; Sidhu et al., 2002). However, neither the precise manner in which PfCRT functions in drug resistance nor its endogenous substrates are known. In the absence of systematic mutagenesis of pfcrt in a controlled genetic background, studies of the contributions of single-point mutations on drug responses have been limited to a small number of changes at position 76 (Cooper et al., 2002; Lakshmanan et al., 2005). Here, we have used single-step drug selection with $\mathrm{QN}$ or $\mathrm{CQ}$ to generate a panel of novel PfCRT mutants. Selection of these mutants provided a unique opportunity to investigate the relationship between drug potency and amino acid changes in previously unexplored domains of PfCRT. Our results indicate that PfCRT residues in positions 72 and 76 of TMD 1 and position 352 of TMD 9 are involved in a drug recognition site critical to the VP-reversible CQR phenotype. In addition to their effect on the CQ phenotype, the physicochemical properties of these key amino acids also affect quantitative responses to other important quinoline antimalarial drugs such as QN and QD. Our study detected no mutations in pfmdr1, whose putative P-glycoprotein-like product (Pgh1) may modulate levels of response to certain DV-targeting drugs (Duraisingh and Cowman, 2005; Sidhu et al., 2005).

In our single-step selection experiments with CQ or QN, the concentration of drug used was rapidly lethal to the drug-sensitive parental $106 / 1^{\mathrm{K} 76}$ or $106 / 1^{761}$ line, preventing proliferation of parasites without adaptive changes. With sufficiently large parasite populations, however, it was possible to select parasite(s) that had incurred a spontaneous point mutation capable of conferring resistance to the media concentration of the drug. Gassis and Rathod (1996) showed that $10^{5}-10^{8}$ parasites are required to select resistance to atovaquone and 5 -fluororotate. In other experiments with $100 \mathrm{nM} \mathrm{CQ}$ pressure on the $106 / 1^{\mathrm{K} 6}$ line, we found that single mutations in pfcrt emerged at a rate of one per $\sim 10^{10}$ drug-sensitive parasites (Cooper et al., 2002). Using single-step drug selection, the probability of simultaneously selecting additional point mutations specifically required for resistance is considered to be multiples of the mutation frequency (Rathod et al., 1997). Although unlikely, we cannot entirely rule out the possible selection of mutations in genes other than pfort and pfmdr1 and their role in the phenotypic changes we observe in our selected lines.

In contrast to these single-step selection experiments, Johnson et al. (2004) used long-term, incremental pressure with halofantrine and amantadine, drugs that show an inverse potency relationship with $C Q$ in $P$. falciparum, to identify new pfcrt mutants from a CQ-resistant line. Amantadine pressure selected a parasite line carrying two PfCRT changes: a novel S163R mutation in TMD 4 and a previously undocumented I356V substitution. Halofantrine pressure also selected S163R, along with two other mutations: T152A and P275L. With the acquisition of amantadine or halofantrine resistance, both lines showed a return to CQS and a loss of the VP-reversible phenotype, changes consistent with gain of a positive charge in the PfCRT pore from the S163R change. The multiple mutations in pfcrt, and perhaps mutations in other genes of these cell lines, may have occurred in a cumulative manner under long-term exposure to sublethal drug concentrations.

In our earlier attempts at single-step CQ selection with the $106 / 1^{\mathrm{K} 76}$ line, we recovered $\mathrm{K} 76 \mathrm{~N}$ and $\mathrm{K} 76 \mathrm{I}$ but not the predominant field K76T mutation (Cooper et al., 2002). K76T was, however, obtained in the experiments reported here, consistent with the probability of its eventual selection in repeated experiments. As expected, the selected $106 / 1^{76 T}$ parasites exhibited a typical VP-reversible phenotype of CQR, and showed a 13-fold increase in the CQ $\mathrm{IC}_{50}$, a 22-fold decrease in cellular accumulation of $\left[{ }^{3} \mathrm{H}\right] \mathrm{CQ}$, and similar expression levels of PfCRT when compared with the $106 / 1^{\mathrm{K} 76}$ parent (Table 1 ; Figs $2 \mathrm{~B}$ and 3). As observed previously, the six PfCRT mutations 
outside of position 76 did not provide any significant increase in the $\mathrm{CQ} I \mathrm{IC}_{50}$ of the $106 / 1^{\mathrm{K} 76}$ parent relative to other CQ-sensitive parasite lines (Cooper et al., 2002). CQR from each of the various $\mathrm{K} 76 \mathrm{~T}, \mathrm{~K} 76 \mathrm{I}$ and $\mathrm{K} 76 \mathrm{~N}$ mutations supports the hypothesis that other PfCRT changes are compensatory mutations and that the loss of the critical charged lysine residue at position 76 dictates the large $I_{50}$ change that results in clinical resistance.

Wild-type PfCRT carries a positively charged lysine at position 76 in TMD 1 predicted to lie near the luminal face of the DV (Fidock et al., 2000; Martin and Kirk, 2004). Passive efflux of $\mathrm{CQ}^{2+}$ out of the DV through wild-type PfCRT may be blocked by a charge-repulsion effect due to the presence of the K76 residue, an idea that has been incorporated into a proposal for resistance termed the 'charged drug leak' model (Warhurst, 2003; Johnson et al., 2004; Bray et al., 2006). In this model, substitution of K76 by a neutral residue $(76 \mathrm{~T}, 76 \mathrm{~N}$ or $76 \mathrm{I})$ could allow exit of $\mathrm{CQ}^{2+}$ out of the DV along its concentration gradient, diminishing availability of drug to bind its haem target, resulting in loss of potency. VP, which selectively restores $\mathrm{CQ}$ potency to resistant parasites, is predicted to bind to the putative PfCRT channel in the vicinity of position 76 . The positive charge carried by VP under the acidic conditions of the DV replaces that found on the wild-type 76 lysine, re-establishing a charge-repulsion effect that retains $C Q$ at its target site (Warhurst et al., 2002). On the other hand, an alternative model of energy-dependent $C Q$ efflux has been supported in reports of $\left[{ }^{3} \mathrm{H}\right] \mathrm{CQ}$ kinetics in natural isolates as well as transformed lines of $P$. falciparum (Krogstad et al., 1992; Sanchez et al., 2004; 2005).

On the controlled genetic background of the $106 / 1^{\mathrm{K} 76}$ line, the relationships of the $76 \mathrm{~K}, 76 \mathrm{~N}, 76 \mathrm{~T}$ and $76 \mathrm{I}$ residues in PfCRT to CQ IC 50 levels suggests an effect of amino acid hydropathy as well as charge in $C Q$ response (Fig. 6A). In the case of $\mathrm{QN}$ or QD, however, the $\mathrm{IC}_{50}$ levels of the $106 / 1^{\mathrm{K} 76}, 106 / 1^{76 \mathrm{~N}}, 106 / 1^{76 \mathrm{l}}$ and $106 / 1^{76 \mathrm{~T}}$ lines (Table 1) do not suggest charge or hydropathy relationships similar to those for $\mathrm{CQ}$. Instead, the increased potency of QN relative to QD against $106 / 1^{761}$ and the effect of VP to increase rather than decrease QN potency (Table 1) points to a complex stereospecific interaction between PfCRT and QN or QD that predominates over a simple weak-base trapping effect.

Stereoselectivity of the cinchona alkaloids against Plasmodium and in other pharmacological systems is well known, highlighting the importance of specific drugreceptor interactions in their activity (Notterman et al., 1986; Sheldon et al., 1990; Wigler and Patterson, 1994; Munoz et al., 1996; Karle and Bhattacharjee, 1999; Hooiveld et al., 2002). A possible explanation for the dramatic increase in QN potency against $106 / 1^{761}$ is a shift to a net inward, as opposed to outward movement of QN through PfCRT (Fig. 7). This explanation is consistent with our

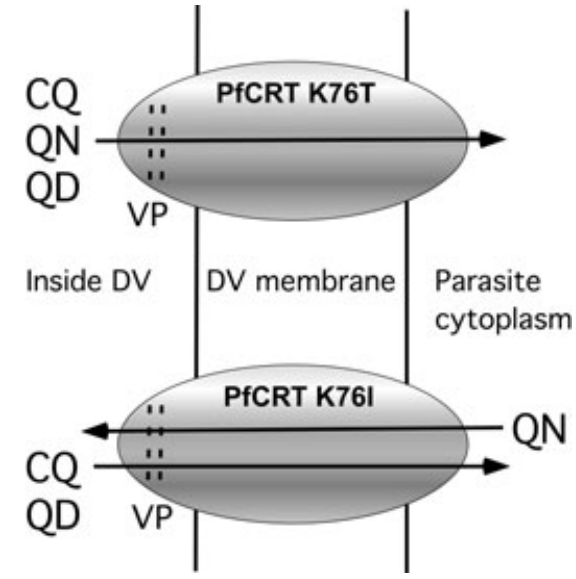

Fig. 7. Illustration of a model of outward and inward movements of QN through different mutant forms of PfCRT. In the CQR form of PfCRT (K76T; top), VP is proposed to bind in the vicinity of the putative PfCRT pore at the inner surface of the DV membrane. When bound, VP may act to reduce active transport or passive efflux of $C Q$ out of the DV, partially restoring $C Q$ sensitivity to resistant lines (Warhurst et al., 2002). In the case of the CQR mutant PfCRT K76I (bottom), however, VP acts oppositely as a competitive inhibitor of QN, although it still potentiates the $C Q$ and QD response in the typical manner. A net inward movement of QN through PfCRT, inhibitable by VP, could account for these observations as well as the hypersensitivity to QN in the $106 / 1^{761}$ line. PfCRT position 76 is predicted to occur in TMD 1 and form part of the protein pore at the interior membrane surface of the DV.

evidence that the K76I mutation leads to an increased cellular QN accumulation but decreased QD accumulation relative to $106 / 1^{\mathrm{K} 76}$ (Cooper et al., 2002). This explanation also accounts for the inhibition rather than chemosensitization of QN activity in $106 / 1^{761}$ parasites by VP (Table 1; Fig. 4A). In accord with an influx of QN through PfCRT, bound VP would inhibit, rather than potentiate the QN response simply by reducing accumulation of QN in the DV. Indeed, analysis of this antagonistic relationship between VP and QN in 106/1 ${ }^{761}$ by Schild regression suggests a simple competitive interaction for a common binding site at PfCRT (Fig. 4B). Additionally, the transition from additivity or antagonism in the $106 / 1^{\mathrm{K} 76}$ and $106 / 1^{76 T}$ lines to strongly synergistic interactions between QN and QD or CQ in 106/1 ${ }^{761}$ attest to a unique interaction between $Q N$ and PfCRT that influences $C Q$ and $Q D$ activity in this line (Fig. 5).

When combined, $C Q$ and $Q N$ have been reported to be antagonistic in $P$. falciparum, both in vitro and in vivo (Hall, 1973; Stahel et al., 1988). In contrast, Wesche and Black (1990) demonstrated that combinations of the cinchona alkaloid diastereomers QN-QD and cinchoninecinchonidine produced an additive effect against $P$. falciparum. In the case of QN and QD, an additive relationship is predicted when two such isomers possess identical mechanisms of action, but differ in their affinity for a common receptor site (Tallarida, 2001). In 106/1 ${ }^{761}$, 
however, a net inward movement of QN through PfCRT may act to reduce efflux of $C Q$ or $Q D$, resulting in high levels of both drugs in the DV and a corresponding synergy. In support of a model for a common drug efflux pathway, Sanchez et al. (2004) demonstrated that QN and QD were able to compete with $C Q$ for transport from the DV by the putative PfCRT efflux pathway. Our data indicate that PfCRT position 76 contains a critical residue affecting interaction with QN and QD, and suggest that the K76I form of PfCRT mediates movement of QN in the opposite direction from QD through discriminating interactions with unique features of each drug's stereoisomeric structure. Careful studies tracking the intracellular movement of radiolabelled $\mathrm{QN}$ and $\mathrm{QD}$ in relation to pfcrt mutations will be required to verify the hypothesis that PfCRT can mediate both the inward and outward movement of drug.

The extreme sensitivity to QN of $106 / 1^{76 I}\left(\mathrm{IC}_{50}=16 \mathrm{nM}\right)$ suggested opportunities for additional informative experiments of single-step selection with QN. Results of these experiments identified three novel amino acid changes in the 106/1 ${ }^{761}$ PfCRT sequence, C72R, Q352K and Q352R, each of which re-introduced a positive charge into a TMD. These changes resulted in increased levels of $C Q$ accumulation, and abrogated the stereoselective potentiation of QN sensitivity by the K76I mutation. In the case of the double mutants, the increase in $C Q$ accumulation was not as dramatic as might be predicted. Only the increased accumulation of CQ by $106 / 1^{761-352 K}$ was significantly different from the $106 / 1^{761}$ parent line (Fig. 3). Nonetheless, a trend was observed between $C Q$ accumulation levels in the double-mutants and their respective $C Q I C_{50}$ values (Table 1, Fig. 3). As these lines were selected for QN resistance rather than $C Q$ resistance, the effect on $C Q$ phenotype in these unusual parasites may involve more than simple changes in drug accumulation levels.

In the case of the QN-selected lines, the positive charge of each mutation probably acts as a second-site suppressor mutation to restore CQS, in agreement with earlier observations on the effect of S163R mutations in PfCRT TMD 4 of resistant $P$. falciparum lines carrying K76T (Johnson et al., 2004). Positions 72, 163 and 352 are found in TMDs 1, 4 and 9, respectively, and their identification as second-site suppressors with respect to the $C Q$ response suggests that they are in or near a drug recognition domain. Our observation that the C72R mutation can compensate for K76 and restore CQS to $106 / 1^{761}$ indicates that 72 and 76 are in proximity to each other within TMD 1, although at slightly different depths within the membrane (Fig. 8). Lakshmanan et al. (2005) have shown that residues 72,74 and 75 are important to the level of VP reversibility of both $C Q$ and $Q N$ in genetically transformed parasites. As pointed out above, the effect of an arginine versus a lysine residue at position 352 in TMD 9 resulted in retention of CQR-like properties in the 106/
$1^{761-352 R}$ line (Table 1). As both mutant lines 106/1 and $106 / 1^{761-352 R}$ differ from $106 / 1^{761}$ by replacement of the polar, uncharged side-chain of glutamine by a positively charged side-chain, the functional chemistry of this residual phenotype is likely to involve structural features of arginine not present in lysine. Relative to lysine, arginine is of greater peptide mass (156 versus 128) and carries a more delocalized positive charge in a complex guanidinium group at the end of a shorter carboncontaining stretch in the side-chain. The amino acid sidechain volume contribution of arginine is consequently only slightly greater than that of lysine (173.4 and 168.6 $\AA^{3}$, respectively; Zamyatnin, 1972) while its hydropathy index suggests an even greater tendency than that of lysine to prefer a polar environment $(-4.5$ versus -3.9 ; Kyte and Doolittle, 1982). Taken together, these results provide strong evidence that $\mathrm{CQ}$ accumulation and susceptibility depend on the presence of a critical positive charge, placed at or near the drug binding site of PfCRT in the form of a lysine or arginine residue in TMD 1, 4 or 9.

Within the drug metabolite effluxer (DME) branch of the DMT superfamily, of which the Plasmodium CRT proteins are predicted members, TMDs 1, 4 and 9 often contain binding motifs required for substrate recognition and translocation (reviewed by Martin and Kirk, 2004). The drug phenotype changes attributable to mutations in these regions of PfCRT are consistent with such findings. To gain further insight into the structural significance of the novel mutations reported here and from other studies, we modelled TMDs 1, 4 and 9 of PfCRT as alpha-helices, representing 3.6 amino acid residues per turn (Fig. 8) (Arai et al., 2004). The wheel projections predict an amphipathic character for the helices, suggesting that they may border a pore in the DV membrane. The polar sides of the helices would form part of a hydrophilic face of the pore, where amino acids 72, 76, 163 and 352 are predicted to occur and likely to interact with drug. In this structural context, mutations affecting the distribution of charge and molecular properties of the pore could determine PfCRT's affinity for drug substrates and possibly their direction of net flux into or out of the DV.

We have demonstrated that $C Q$ susceptibility is related to charge changes within TMD 1,4 or 9 , as well as hydropathy of the substituent amino acid at position 76 . Although the data do not necessarily discriminate between active or passive efflux mechanisms, they do indicate that direct PfCRT-drug interactions are critical to the loss of $C Q$ from the DV. With respect to $Q N$ and $Q D$ susceptibilities, however, the evidence for drug binding appears more consistent with a selective transport process as opposed to a passive leak model based on charge loss or gain mutations. In either scenario the activity of PfCRT appears to be highly dependent on drug structure, as the susceptibility changes to $C Q, Q N$ and 


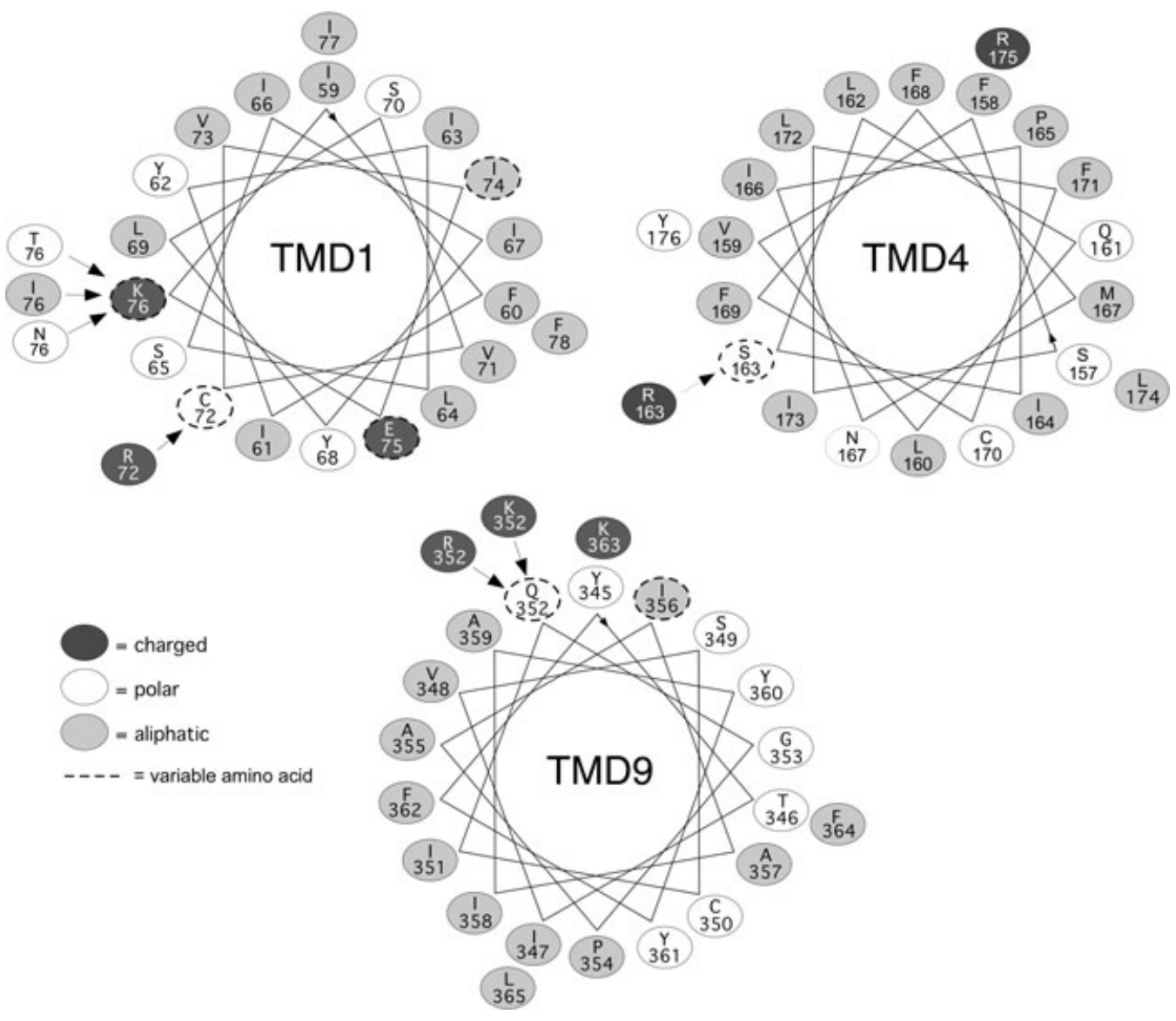

Fig. 8. Helical wheel projections of PfCRT TMDs 1, 4 and 9. Projections of TMDs 1, 4 and 9 predict the amphipathic nature (a predominantly polar and hydrophobic side) of the membrane spanning alpha-helices. In each case, the charged residues associated with CQ response lie in the polar region of the helices, suggesting the polar faces may be oriented towards a pore or channel where they interact with substrates and drugs. The hydrophobic faces may be associated with membrane lipid or other apolar surfaces. Residue numbers correspond to the positions of residues in relation to the outside surface of the DV membrane. Arrows indicate alternative mutations associated with the CQ response.

The S163R mutation in TMD 4 was reported by Johnson et al. (2004).

QD are distinct. While our results implicate a critical role for PfCRT-drug interactions in quinoline susceptibility, additional studies will be required to determine the exact mechanism by which PfCRT operates in drug flux.

\section{Experimental procedures}

\section{Plasmodium falciparum culture}

Parasites were grown in $\mathrm{AB}^{+}$or $\mathrm{O}^{+}$human red blood cells using RPMI 1640 supplemented with $0.5 \%$ Albumax I (Invitrogen, Carlsbad, CA), $0.25 \%$ sodium bicarbonate and $0.01 \mathrm{mg} \mathrm{ml}^{-1}$ gentamicin under an atmosphere of $90 \%$ nitrogen, $5 \%$ oxygen and $5 \%$ carbon dioxide. Parasites synchrony was maintained by treatment with $5 \%$ sorbitol solution (Lambros and Vanderberg, 1979).

\section{Single-step selection for $C Q$ and $Q N$ resistance}

Single-step selection was carried out based on the methods of Rathod et al. (1997) and Cooper et al. (2002). Parasites of the $106 / 1^{\mathrm{K} 76}$ or $106 / 1^{761}$ line were initially grown under normal culture conditions, at $5 \%$ haematocrit, without the presence of drug. When a $50 \mathrm{ml}$ volume of culture reached a parasitaemia of $\sim 5 \%$, it was split equally into four flasks, and restored to a volume of $50 \mathrm{ml}$ and haematocrit of $5 \%$ with fresh red blood cells and media. Cultures were maintained until again reaching $\sim 5 \%$ parasitaemia (representing $\sim 10^{9}$ parasites per flask), at which time either $100 \mathrm{nM} \mathrm{CQ} \mathrm{(106/}$ $1^{\mathrm{K} 76}$ ) or QN $\left(106 / 1^{761}\right)$ was added. This concentration was sufficient to rapidly kill any parasites except a possible preexisting, drug-resistant mutant. Drug-containing medium was replaced daily for the first week. At 7 days post drug, $50 \%$ of the remaining red blood cells were replaced with fresh cells. Following day 7 , culture medium was replaced every third day with fresh drug-media, at which time cultures were monitored for the presence of any emergent parasites by examining Giemsa-stained thin films. Fifty per cent of the red blood cells were replaced at every other medium change until the end of the experiment. In all cases, parallel, drug-free cultures were maintained to monitor laboratory culture conditions. Drug-pressured cultures were monitored for 60 days or until living parasites were observed. Emergent 
parasites from any positive flask were cloned by limiting dilution (Rosario, 1981). Clonal parasites were processed for genomic DNA preparations, cryopreservation and grown for antimalarial dose-response assays.

\section{Microsatellite genotyping and sequencing of pfcrt and pfmdr1}

The entire open reading frame sequences of pfcrt and pfmdr1 were amplified from genomic DNA. After treatment with ExoSAP-IT (US Biochemical, Cleveland $\mathrm{OH}$ ), polymerase chain reaction products were directly sequenced on an $A B I$ 3730XL automatic DNA analyser (Applied Biosystems, Foster City, CA) using specific internal primers (Mu et al., 2003). DNA from parasite clones was genotyped using the multicopy microsatellite marker PfRRM as described. (Su et al., 1998; Vieira et al., 2004). Electropherograms were analysed using the GeneScan 3.7 software package (Applied Biosystems).

\section{SDS-PAGE and immunoblotting}

Crude membrane fractions of trophozoite-stage parasites for SDS-PAGE were prepared from synchronous cultures. Trophozoites were isolated from red blood cells using $0.1 \%$ saponin. Parasite pellets were freeze-thawed three times, after which the insoluble portion was pelleted by centrifugation at $14 \mathrm{~K}$ r.p.m. for $10 \mathrm{~min}$. Pellets were solubilized with reducing SDS-PAGE loading buffer and heated to $95^{\circ} \mathrm{C}$ for $5 \mathrm{~min}$. Protein samples representing $\sim 4 \times 10^{6}$ trophozoites were separated on $12 \%$ polyacrylamide gels and transferred to PVDF membrane. Membranes were incubated with affinitypurified anti-PfCRT polyclonal antisera (anti-PfCRT K IgG; Fidock et al., 2000) or anti- $\alpha$-tubulin II antibody (MRA-37 MR4, Manassas, VA) (Rohrbach et al., 2006) diluted 1:1000 overnight at $5^{\circ} \mathrm{C}$. Proteins were detected by chemiluminescence (Pierce Biotechnology, Rockford, IL) following incubation with rabbit horseradish peroxidase-conjugated secondary antibody (Jackson ImmunoResearch, West Grove, PA).

\section{Drug susceptibility testing}

The in vitro antiparasitic effect of the various drugs was measured by $\left[{ }^{3} \mathrm{H}\right]$-hypoxanthine (PerkinEImer Life Sciences, Boston, MA) incorporation (Desjardins et al., 1979; Ferdig et al., 2004). Per cent inhibition of growth was plotted against log drug concentration to generate dose-response curves. The half-maximal inhibitory response $\left(\mathrm{IC}_{50}\right)$, defined as the drug concentration at which growth was inhibited by $50 \%$, was estimated by curve-fitting the response data with a variable-slope sigmoidal function (Prism 4.0c; GraphPad Software, San Diego, CA). VP was used at a fixed concentration of $0.9 \mu \mathrm{M}$ in reversibility assays. Dose-response curves represent 3-18 independent assays. Relevant $\mathrm{IC}_{50}$ values from single-drug assays were tested for statistical significance by comparing mean values from each mutant line to that of its parent line by an unpaired $t$-test $(P<0.05)$. Mean Hill slope values between parasite lines were tested for statistical significance using an $F$-test $(P<0.05)$.

For drug combination testing, drugs were mixed in volumetric ratios of $9: 1,7: 3,1: 1,3: 7$ and 1:9. The starting unmixed drug concentrations, CQ $2.5 \mu \mathrm{M}$; QN $2.5 \mu \mathrm{M}$; QD $625 \mathrm{nM}$, were determined from single-drug assays that allowed the $\mathrm{IC}_{50}$ to be centred in the test plate. Single drugs and drug combinations were serially diluted twofold across the 96-well plate prior to adding parasite culture. Results from the dose-response curves were expressed as sums of the fractional $\mathrm{IC}_{50}$ (FIC) values and plotted as isobolograms (Berenbaum, 1978). FIC values $<1$ indicate synergism, those equal to 1 indicate additivity and those $>1$ indicate antagonism. Experiments were repeated on three independent occasions, with data from all experiments shown on the plots.

To determine the mechanism of QN inhibition by VP in the $106 / 1^{761}$ line, Schild analysis was performed (Arunlakshana and Schild, 1959). A series of QN dose-response curves was generated in the presence and absence of fixed concentrations of VP and fit by non-linear regression. Data derived from the dose-response curves were plotted as log(dose ratio -1) as a function of the log VP concentration. Dose ratio was defined as the equi-active concentration of $\mathrm{QN}$ in the presence of VP divided by the concentration of QN in the absence of VP. Linear regression was performed to derive the Schild regression and provide an estimate of the $K_{\mathrm{B}}$ for VP binding. Data were derived from a total of three independent tests of the QN with VP dose-response series.

\section{$\left[{ }^{3} H\right] C Q$ accumulation assays}

Chloroquine accumulation was determined by incubating trophozoite-infected erythrocytes with $50 \mathrm{nM}\left[{ }^{3} \mathrm{H}\right] \mathrm{CQ}$ (Amersham Biosciences, Piscataway, $\mathrm{NJ}$ ) and processed as described (Cooper et al., 2002). Parasites were collected at 0 and 60 min for $\left[{ }^{3} \mathrm{H}\right] \mathrm{CQ}$ uptake measurements. Cellular accumulation ratio (CAR) was determined by dividing the amount of $\left[{ }^{3} \mathrm{H}\right] \mathrm{CQ}$ measured in infected erythrocytes by the $\left[{ }^{3} \mathrm{H}\right] \mathrm{CQ}$ measured in a similar number of uninfected erythrocytes. Accumulation experiments were conducted on three to four independent occasions, with each assay conducted in duplicate. A $t$-test was performed to determine whether mean CAR values between relevant parasite lines were significantly different $(P<0.05)$.

\section{Correlation of amino acid physicochemical properties with drug response}

Hydrophobicity values were from the consensus scale of Kyte and Doolittle (1982). Side-chain volumes were from Zamyatnin (1972). Physical parameters for each of the four different amino acids at PfCRT position $76(\mathrm{~K}, \mathrm{~N}, \mathrm{~T}, \mathrm{I})$ were plotted versus the $C Q I C_{50}$ values. Linear regression analysis was performed using Prism 4 software. Slope values were tested for statistical differences from zero $(P<0.05)$.

\section{Secondary structure predictions}

Putative transmembrane domains and helical projections were derived using ConPred II (http://bioinfo.si.hirosaki-u.ac. $\mathrm{jp} / \sim$ ConPred2/), a web-based algorithm for predicting secondary structure of transmembrane proteins. Conpred II is a consensus method that utilizes multiple topology prediction methods (Arai et al., 2004). 


\section{Acknowledgements}

The authors thank Dayani Tennakoon and Heidi Schmitt for technical assistance, and Paul Roepe for valuable discussions. R.A.C. is supported by NIH Grant Al055601. M.T.F., B.D. and J.J.P. are supported by NIH Grant AI055035. This research was also supported in part by the Intramural Research Program of the NIH, NIAID. J. Mu, X-Z. Su and T.E. Wellems are Federal Government employees. No claim to original US government works.

\section{References}

Arai, M., Mitsuke, H., Ikeda, M., Xia, J.X., Kikuchi, T., Satake, M., and Shimizu, T. (2004) ConPred II: a consensus prediction method for obtaining transmembrane topology models with high reliability. Nucleic Acids Res 32: 390393.

Arunlakshana, O., and Schild, H.O. (1959) Some quantitative uses of drug antagonists. Br J Pharmacol Chemother 14: 48-58.

Baird, J.K. (2005) Effectiveness of antimalarial drugs. N Engl J Med 352: 1565-1577.

Berenbaum, M.C. (1978) A method for testing for synergy with any number of agents. J Infect Dis 137: 122-130.

Bray, P.G., Mungthin, M., Ridley, R.G., and Ward, S.A. (1998) Access to hematin: the basis of chloroquine resistance. Mol Pharmacol 54: 170-179.

Bray, P.G., Martin, R.E., Tilley, L., Ward, S.A., Kirk, K., and Fidock, D.A. (2005) Defining the role of PfCRT in Plasmodium falciparum chloroquine resistance. Mol Microbiol 56: 323-333.

Bray, P.G., Mungthin, M., Hastings, I.M., Biagini, G.A., Saidu, D.K., Lakshmanan, V., et al. (2006) PfCRT and the transvacuolar proton electrochemical gradient: regulating the access of chloroquine to ferriprotoporphyrin IX. Mol Microbiol 62: 238-251.

Chou, A.C., and Fitch, C.D. (1980) Hemolysis of mouse erythrocytes by ferriprotoporphyrin IX and chloroquine. Chemotherapeutic implications. J Clin Invest 66: 856-858.

Cooper, R.A., Ferdig, M.T., Su, X.Z., Ursos, L.M., Mu, J., Nomura, T., et al. (2002) Alternative mutations at position 76 of the vacuolar transmembrane protein PfCRT are associated with chloroquine resistance and unique stereospecific quinine and quinidine responses in Plasmodium falciparum. Mol Pharmacol 61: 35-42.

Cooper, R.A., Hartwig, C.L., and Ferdig, M.T. (2005) pfcrt is more than the Plasmodium falciparum chloroquine resistance gene: a functional and evolutionary perspective. Acta Trop 94: 170-180.

Desjardins, R.E., Canfield, C.J., Haynes, J.D., and Chulay, J.D. (1979) Quantitative assessment of antimalarial activity in vitro by a semiautomated microdilution technique. Antimicrob Agents Chemother 16: 710-718.

Djimde, A., Doumbo, O.K., Cortese, J.F., Kayentao, K., Doumbo, S., Diourte, Y., et al. (2001) A molecular marker for chloroquine-resistant falciparum malaria. $N$ Engl J Med 344: 257-263.

Dorn, A., Vippagunta, S.R., Matile, H., Jaquet, C., Vennerstrom, J.L., and Ridley, R.G. (1998) An assessment of drug-haematin binding as a mechanism for inhibition of haematin polymerisation by quinoline antimalarials. Biochem Pharmacol 55: 727-736.

Duraisingh, M.T., and Cowman, A.F. (2005) Contribution of the pfmdr1 gene to antimalarial drug-resistance. Acta Trop 94: 181-190.

Durrand, V., Berry, A., Sem, R., Glaziou, P., Beaudou, J., and Fandeur, T. (2004) Variations in the sequence and expression of the Plasmodium falciparum chloroquine resistance transporter (PfCRT) and their relationship to chloroquine resistance in vitro. Mol Biochem Parasitol 136: 273-285.

Ferdig, M.T., Cooper, R.A., Mu, J., Deng, B., Joy, D.A., Su, X.Z., and Wellems, T.E. (2004) Dissecting the loci of lowlevel quinine resistance in malaria parasites. Mol Microbiol 52: 985-997.

Fidock, D.A., Nomura, T., Talley, A.K., Cooper, R.A., Dzekunov, S.M., Ferdig, M.T., et al. (2000) Mutations in the $P$. falciparum digestive vacuole transmembrane protein PfCRT and evidence for their role in chloroquine resistance. Mol Cell 6: 861-871.

Fitch, C.D. (2004) Ferriprotoporphyrin IX, phospholipids, and the antimalarial actions of quinoline drugs. Life Sci 74: 1957-1972.

Gassis, S., and Rathod, P.K. (1996) Frequency of drug resistance in Plasmodium falciparum: a nonsynergistic combination of 5-fluoroorotate and atovaquone suppresses in vitro resistance. Antimicrob Agents Chemother 40: 914-919.

Goldberg, D.E., Slater, A.F., Cerami, A., and Henderson, G.B. (1990) Hemoglobin degradation in the malaria parasite Plasmodium falciparum: an ordered process in a unique organelle. Proc Natl Acad Sci USA 87: 2931-2935.

Hall, A.P. (1973) Letter: quinine and chloroquine antagonism in falciparum malaria. Trans R Soc Trop Med Hyg 67: 425.

Hooiveld, G.J., Heegsma, J., van Montfoort, J.E., Jansen, P.L., Meijer, D.K., and Muller, M. (2002) Stereoselective transport of hydrophilic quaternary drugs by human MDR1 and rat Mdr1b P-glycoproteins. Br J Pharmacol 135: 16851694.

Huaman, M.C., Yoshinaga, K., Suryanatha, A., Suarsana, N., and Kanbara, H. (2004) Short report: polymorphisms in the chloroquine resistance transporter gene in Plasmodium falciparum isolates from Lombok, Indonesia. Am J Trop Med Hyg 71: 40-42.

Johnson, D.J., Fidock, D.A., Mungthin, M., Lakshmanan, V., Sidhu, A.B., Bray, P.G., and Ward, S.A. (2004) Evidence for a central role for PfCRT in conferring Plasmodium falciparum resistance to diverse antimalarial agents. Mol Cell 15: 867-877.

Karle, J.M., and Bhattacharjee, A.K. (1999) Stereoelectronic features of the cinchona alkaloids determine their differential antimalarial activity. Bioorg Med Chem 7: 1769-1774.

Krogstad, D.J., Gluzman, I.Y., Kyle, D.E., Oduola, A.M., Martin, S.K., Milhous, W.K., and Schlesinger, P.H. (1987) Efflux of chloroquine from Plasmodium falciparum: mechanism of chloroquine resistance. Science 238: 1283-1285.

Krogstad, D.J., Gluzman, I.Y., Herwaldt, B.L., Schlesinger, P.H., and Wellems, T.E. (1992) Energy dependence of chloroquine accumulation and chloroquine efflux in Plasmodium falciparum. Biochem Pharmacol 43: 57-62.

Kyle, D.E., Oduola, A.M., Martin, S.K., and Milhous, W.K. (1990) Plasmodium falciparum: modulation by calcium antagonists of resistance to chloroquine, desethylchloro- 
quine, quinine, and quinidine in vitro. Trans $R$ Soc Trop Med Hyg 84: 474-478.

Kyte, J., and Doolittle, R.F. (1982) A simple method for displaying the hydropathic character of a protein. $\mathrm{J} \mathrm{Mol} \mathrm{Biol}$ 157: 105-132.

Laduron, P.M. (1988) Stereospecificity in binding studies. A useful criterion though insufficient to prove the presence of receptors. Biochem Pharmacol 37: 37-40.

Lakshmanan, V., Bray, P.G., Verdier-Pinard, D., Johnson, D.J., Horrocks, P., Muhle, R.A., et al. (2005) A critical role for PfCRT K76T in Plasmodium falciparum verapamilreversible chloroquine resistance. EMBO J 24: 22942305.

Lambros, C., and Vanderberg, J.P. (1979) Synchronization of Plasmodium falciparum erythrocytic stages in culture. J Parasitol 65: 418-420.

Martin, R.E., and Kirk, K. (2004) The malaria parasite's chloroquine resistance transporter is a member of the drug/ metabolite transporter superfamily. Mol Biol Evol 21: 1938-1949.

Mu, J., Ferdig, M.T., Feng, X., Joy, D.A., Duan, J., Furuya, T., et al. (2003) Multiple transporters associated with malaria parasite responses to chloroquine and quinine. Mol Microbiol 49: 977-989.

Munoz, R., Garcia, E., and De la Campa, A.G. (1996) Quinine specifically inhibits the proteolipid subunit of the $F_{0} F_{1}$ $\mathrm{H}^{+}$-ATPase of Streptococcus pneumoniae. J Bacteriol 178: 2455-2458.

Naude, B., Brzostowski, J.A., Kimmel, A.R., and Wellems, T.E. (2005) Dictyostelium discoideum expresses a malaria chloroquine resistance mechanism upon transfection with mutant, but not wild-type, Plasmodium falciparum transporter PfCRT. J Biol Chem 280: 2559625603.

Notterman, D.A., Drayer, D.E., Metakis, L., and Reidenberg, M.M. (1986) Stereoselective renal tubular secretion of quinidine and quinine. Clin Pharmacol Ther 40: 511-517.

Pagola, S., Stephens, P.W., Bohle, D.S., Kosar, A.D., and Madsen, S.K. (2000) The structure of malaria pigment beta-haematin. Nature 404: 307-310.

Rathod, P.K., McErlean, T., and Lee, P.C. (1997) Variations in frequencies of drug resistance in Plasmodium falciparum. Proc Natl Acad Sci USA 94: 9389-9393.

Rohrbach, P., Sanchez, C.P., Hayton, K., Friedrich, O., Patel, J., Sidhu, A.B., et al. (2006) Genetic linkage of pfmdr1 with food vacuolar solute import in Plasmodium falciparum. EMBO J 25: 3000-3011.

Rosario, V. (1981) Cloning of naturally occurring mixed infections of malaria parasites. Science 212: 1037-1038.

Saliba, K.J., Folb, P.I., and Smith, P.J. (1998) Role for the Plasmodium falciparum digestive vacuole in chloroquine resistance. Biochem Pharmacol 56: 313-320.

Sanchez, C.P., McLean, J.E., Stein, W., and Lanzer, M. (2004) Evidence for a substrate specific and inhibitable drug efflux system in chloroquine resistant Plasmodium falciparum strains. Biochemistry 43: 16365-16373.

Sanchez, C.P., McLean, J.E., Rohrbach, P., Fidock, D.A., Stein, W.D., and Lanzer, M. (2005) Evidence for a pfcrtassociated chloroquine efflux system in the human malarial parasite Plasmodium falciparum. Biochemistry 44: 98629870.
Sheldon, R.S., Rahmberg, M., and Duff, H.J. (1990) Quinidine/quinine: stereospecific electrophysiologic and antiarrhythmic effects in a canine model of ventricular tachycardia. J Cardiovasc Pharmacol 16: 818-823.

Sidhu, A.B., Verdier-Pinard, D., and Fidock, D.A. (2002) Chloroquine resistance in Plasmodium falciparum malaria parasites conferred by pfcrt mutations. Science 298: 210213.

Sidhu, A.B., Valderramos, S.G., and Fidock, D.A. (2005) pfmdr1 mutations contribute to quinine resistance and enhance mefloquine and artemisinin sensitivity in Plasmodium falciparum. Mol Microbiol 57: 913-926.

Stahel, E., Druilhe, P., and Gentilini, M. (1988) Antagonism of chloroquine with other antimalarials. Trans $R$ Soc Trop Med Hyg 82: 221.

Su, X.Z., Carucci, D.J., and Wellems, T.E. (1998) Plasmodium falciparum: parasite typing by using a multicopy microsatellite marker, PfRRM. Exp Parasitol 89: 262-265.

Sullivan, D.J., Jr, Gluzman, I.Y., Russell, D.G., and Goldberg, D.E. (1996) On the molecular mechanism of chloroquine's antimalarial action. Proc Natl Acad Sci USA 93: 1186511870.

Tallarida, R.J. (2001) Drug synergism: its detection and applications. J Pharmacol Exp Ther 298: 865-872.

Tran, C.V., and Saier, M.H., Jr (2004) The principal chloroquine resistance protein of Plasmodium falciparum is a member of the drug/metabolite transporter superfamily. Microbiology 150: 1-3.

Triggle, D.J. (1996) Ion channels as pharmacologic receptors: the chirality of drug interactions. Chirality 8: 35-38.

Vieira, P.P., Ferreira, M.U., Das Gracas Alecrim, M., Alecrim, W.D., Da Silva, L.H., Sihuincha, M.M., et al. (2004) pfcrt polymorphism and the spread of chloroquine resistance in Plasmodium falciparum populations across the Amazon Basin. J Infect Dis 190: 417-424.

Warhurst, D.C. (2003) Polymorphism in the Plasmodium falciparum chloroquine-resistance transporter protein links verapamil enhancement of chloroquine sensitivity with the clinical efficacy of amodiaquine. Malar J 2: 31.

Warhurst, D.C., Craig, J.C., and Adagu, I.S. (2002) Lysosomes and drug resistance in malaria. Lancet 360: 15271529.

Wesche, D.L., and Black, J. (1990) A comparison of the antimalarial activity of the cinchona alkaloids against Plasmodium falciparum in vitro. J Trop Med Hyg 93: 153-159.

Wigler, P.W., and Patterson, F.K. (1994) Reversal agent inhibition of the multidrug resistance pump in human leukemic lymphoblasts. Biochim Biophys Acta 1189: 1-6.

Wootton, J.C., Feng, X., Ferdig, M.T., Cooper, R.A., Mu, J., Baruch, D.I., et al. (2002) Genetic diversity and chloroquine selective sweeps in Plasmodium falciparum. Nature 418: 320-323.

Yayon, A., Cabantchik, Z.I., and Ginsburg, H. (1985) Susceptibility of human malaria parasites to chloroquine is $\mathrm{pH}$ dependent. Proc Natl Acad Sci USA 82: 2784-2788.

Zamyatnin, A.A. (1972) Protein volume in solution. Prog Biophys Mol Biol 24: 107-123.

Zhang, H., Paguio, M., and Roepe, P.D. (2004) The antimalarial drug resistance protein Plasmodium falciparum chloroquine resistance transporter binds chloroquine. Biochemistry 43: 8290-8296. 